\title{
Test phytochimique et insecticide de trois extraits organiques de feuilles de Ficus thonningii sur Callosobruchus maculatus Fabricius
}

\author{
El Hadji Gorgui DIOUF ${ }^{1 *}$, Abdoulaye SAMB ${ }^{1}$, Oumar SYLLA ${ }^{3}$, Aden Elmi KAFIA ${ }^{3}$, \\ Moussoukhoye DIOP ${ }^{1}$, Dogo SECK $^{2}$ et Koffi NGUESSAN ${ }^{4}$

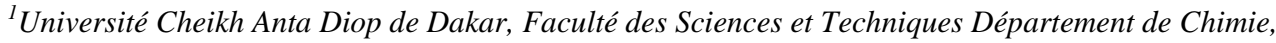 \\ Laboratoire des Produits Naturels, Dakar, Sénégal. \\ ${ }^{2}$ Centre Régionale de Recherche en Écotoxicologie et Sécurité Environnementale \\ (CERES-Locustox) Route de Rufisque, km 15. BP 3300. SN-Dakar, Sénégal. \\ ${ }^{3}$ Faculté de Médicine, de Pharmacie ET d'Odontostomatologie, Département des Sciences Pharmaceutiques, \\ Physiques et Chimiques, Service de Toxicologie et d'Hydrologie, Sénégal. \\ ${ }^{4}$ Université de Cocody-Abidjan, UFR: Biosciences, Laboratoire de Botanique. 22, \\ BP: 582 Abidjan 22, Côte-d'Ivoire. \\ *Auteur correspondant; E-mail :goorgui82@yahoo.fr ; Tel : +221 773675508
}

\section{RESUME}

Pour trouver des alternatifs aux insecticides de synthèse, les extraits de plantes sont de plus en plus utilisés par les paysans pour protéger les stocks de récolte contre les insectes ravageurs. Ainsi, des tests phytochimiques et insecticides respectivement par chromatographie sur couche mince et par contact sont effectués sur trois extraits organiques (cyclohexanique, chloroformique et méthanolique) de Ficus thonningii. Les données ont été analysées par la procédure General Linear Model à l'aide du logiciel Minitab 17. Les facteurs étudiés sont : le temps, le nombre d'insectes morts et le nombre d'insectes émergés, ainsi que leurs interactions. Les résultats de l'analyse statistique ont montré que l'extrait méthanolique donne un meilleur taux de mortalité sur Callosobruchus maculatus aux dates $3^{\text {ème }}$ jour, $5^{\mathrm{e}}$ jour, $6^{\mathrm{e}}$ jour, $7^{\mathrm{e}}$ jour et $8^{\text {ème }}$ jour. Ces résultats sont corroborés par les tests phytochimiques avec l'identification de molécules (alcaloïdes, flavanoïdes, tanins, polyphénols et saponosides...) susceptibles d'être responsables de cette activité insecticide.

(C) 2014 International Formulae Group. All rights reserved.

Mots clés: Extraits, Ficus thonningii, Callosobruchus maculatus, niébé.

\section{INTRODUCTION}

Le niébé est une importante denrée de base en Afrique subsaharienne, particulièrement dans les savanes arides de l'Afrique de l'Ouest. Ses graines représentent une précieuse source de protéines végétales, de vitamines et de revenus pour l'homme, ainsi que de fourrage pour les animaux. Les feuilles juvéniles et les gousses immatures sont consommées sous forme de légume (IITA, 2009).
La superficie annuelle cultivée dans le monde s'élève à plus de 12,5 millions d'ha, dont 9,8 millions sont réalisés en Afrique de l'Ouest, qui est la plus grande zone de production et de consommation du niébé dans le monde (Singh et al., 1997; CGIAR, 2001; FAOSTAT, 2004). Le centième des superficies emblavées en Afrique de l'Ouest revient au Sénégal, faisant de ce pays le cinquième producteur africain après le Nigeria, le Niger, le Mali et le Burkina Faso 
(Cissé et Hall, 2003; DSDIA/DAPS/MAE, 1999 à 2003; Nkouannessi, 2005). Ainsi, la culture du niébé occupe une place importante au Sénégal.

Nonobstant son importance, les rendements du niébé sont inférieurs dans certaines régions à la moyenne de l'Afrique qui est de $700 \mathrm{~kg} / \mathrm{ha}$ (Kouakou et al., 2007). Parmi les contraintes à la production du niébé, les insectes ravageurs causent le plus grand problème au niébé. En effet, le niébé est attaqué par un large spectre de ravageurs depuis la germination jusqu'à la récolte. Dans la gamme très large de ces insectes ravageurs du niébé, les coléoptères Bruchidae dont notamment Callosobruchus maculatus Fabricius sont parmi les plus redoutables du fait que leurs attaques débutent au champ, pour s'étendre ensuite à l'entrepôt où la population de bruches peut croître rapidement (Djossou, 2006).

Pour lutter contre les insectes ravageurs de stock de récolte, la méthode la plus utilisée est l'usage d'insecticides de synthèse (carbamates, organophosphorés, organochlorés, pyréthrinoïdes, ...). Ces pesticides de longue persistance assurent la protection des semences depuis les magasins de stockage jusque dans les champs après les semis ainsi que des jeunes plantules contre les insectes et les maladies (Djossou, 2006). Ces méthodes utilisées pour limiter les pertes dans les stocks peuvent induire une intoxication chronique des consommateurs, une résistance chez les ravageurs et avoir un impact négatif sur l'environnement (Ngamo \& Hance, 2007). Les insecticides posent en outre, des problèmes de disponibilité, de stockage et de coût. Dans la recherche de méthodes alternatives de lutte, le règne végétal offre beaucoup de possibilités. Des travaux sont effectués dans ce contexte et ont montré une efficacité des extraits des plantes. En effet, les plantes constituent une source de substances naturelles qui présente un grand potentiel d'application contre les insectes et d'autres parasites des plantes et du monde animal (Bouzouita et al., 2008).
L'objectif de ce travail est d'étudier l'activité insecticide de trois extraits organiques (cyclohexanique, chlroformique et méthanolique) de Ficus thonningii sur Callosobruchus maculatus d'une part. D'autre part, il s'agira d'identifier les différentes familles de composés chimiques présentes dans ces trois extraits.

\section{MATERIEL ET METHODES \\ Matériel \\ Matériel végétal}

Le matériel végétal était constitué de feuilles et d'écorces de tige de Ficus thonningii. Elles ont été broyées et utilisées pour les extractions avec des solvants de gradient de polarités croissantes.

Solvants

Les différents solvants utilisés pour l'extraction, les tests d'identification, les tests biologiques et les CCM sont :

l'Acétate d'éthyle PA-ACS-ISO; Mininum essai (G.C.): 99, 5\%; Identité: IR p/t.; Densité à 20/4: 0, 9000-0,902;

l'Acide acétique PA-ACS-ISO; Mininum essai (G.C.): 99, 5\%; Identité: IR p/t.; Densité à 20/4: 1, 05;

Cyclohexane, (Reag. USP, Ph.Eur.) PA-ACS; Mininum essai (G.C.): 99, 0\%; Identité: IR p/t.; Densité à 20/20: 0, 659-0, 663 ;

- Chloroforme (Reag. USP, Ph.Eur.)PA-ACS-ISO; Mininum essai (G.C.): 99, 5\%; Identité: IR p/t.; Densité à 20/4: 1, 48; - Dichmorométhane stabilize avec amylene PA-ACS-ISO; Mininum essai (G.C.): 99,5\%; Identité: IR pt.; Densité à 20/4: 1, 323-1, 325;

- Methanol (Reag. USP, Ph.Eur.)PAACS-ISO; Mininum essai (G.C.): 99, 5\%; Identité: IR p/t.; Densité à 20/4: 0, 791-0, 792. Réactifs

Pour l'identification et la mise en évidences des différents groupes chimiques présents dans chaque extrait, plusieurs types de réactifs et témoins ont été utilisés.

Pour les tanins, l'Acide tannique a été utilisé comme témoin et le Chlorure ferrique à $20 \%$ a servi de révélateur. 
Pour la recherche de flavonoïdes et des polyphénols, le témoin est le Vitexine et pour le réactif le Chlorure d'Aluminium a été utilisé.

Les Alcaloïdes ont été révélés grâce au réactif de Draggendorf en utilisant comme témoin le Cinchonine. Le réactif de Draggendorf est préparé à partir d'une solution composée de $0,85 \mathrm{~g}$ de nitrate basique de bismuth et $10 \mathrm{~g}$ d'acide tartrique dans $40 \mathrm{ml}$ d'eau (solution A) et une solution contenant $16 \mathrm{~g}$ de $\mathrm{KI}$ dans $40 \mathrm{ml}$ d'eau (solution B). Mélanger extemporanément $5 \mathrm{ml}$ de A, $5 \mathrm{ml}$ de B, $100 \mathrm{ml}$ d'eau et $20 \mathrm{~g}$ d'acide tartrique.

\section{Matériel animal}

Le matériel animal concerne l'espèce Callosobruchus maculatus obtenus par élevage de masse.

\section{Méthodes \\ Récolte et séchage}

Les spécimens sont récoltés dans la communauté rurale de Keur Balla, localité située dans le département de Mbour qui est une subvention de la région de Thiès comprise entre les latitudes $14^{\circ} 02^{\prime}$ et $15^{\circ} 27^{\prime}$ Nord et les longitudes $16^{\circ} 09^{\prime}$ et $17^{\circ} 12^{\prime}$ Ouest. Ainsi, grâce à l'aide d'un vieux tradipraticien, nous avons récolté les feuilles et tiges de Ficus thonningii. Le matériel végétal obtenu a été séché à l'ombre à l'abri du solaire pendant deux semaines.

\section{Extraction}

La technique utilisée pour les extractions est la macération durant 24 heures avec des solvants de gradient de polarité croissante (Cyclohexane, Chloroforme et Méthanol). L'extrait obtenu est concentré à l'aide d'un évaporateur rotatif pendant 15 minutes avant d'être séché à la température ambiante $\left(25^{\circ} \mathrm{C}\right)$ et à l'abri du soleil durant 24 à 48 heures.

\section{Les élevages de masse}

Les élevages de masse concernent l'espèce Callosobruchus maculatus. Du niébé infesté a été trouvé au marché sur lequel des tris ont été opérés pour récupérer des insectes et lancer les élevages de masse. Les élevages sont lancés dans des bocaux en verre de 500 $\mathrm{mL}$ de volume environ. A l'intérieur de chaque bocal, 20 à 25 insectes sont introduits et du coton hydrophile imprégné d'eau pour créer les conditions d'humidité nécessaire pour une bonne reproduction des insectes. Ces bocaux sont perforés et recouverts de toile de moustiquaire pour permettre aux insectes de respirer. Les élevages sont faits à l'ombre et à la température ambiante $\left(25^{\circ} \mathrm{C}\right.$ environ $)$.

$\mathrm{Au}$ bout de 17 à 20 jours, nous avons observé des émergences. Les tests d'activité insecticide sont effectués sur des insectes de première génération c'est-à-dire qui sont âgés entre 0 et 24 heures.

\section{Identification des principes actifs}

Pour l'identification des différents groupes chimiques par chromatographie sur couche mince (CCM), la méthode décrite par Bassène (2014) a été utilisée.

La mise en évidence des différentes familles de composés chimiques présentes dans Ficus thonningii a été faite par Chromatographie sur Couche Mince (CCM) et par des tests de coloration et de précipitation.

\section{CCM des alcaloüdes}

Pour l'identification des alcaloïdes, le gel de Silice est utilisé comme phase stationnaire. L'éluant était un mélange de Chloroforme plus diéthylamine $(45 \mathrm{~V} / 5 \mathrm{~V})$. Le témoin utilisé est le Cinchonine. La révélation était faite avec le réactif de Draggendorf. La coloration rouge et rouge orangée marque la présence d'alcaloïdes dans les extraits. Le développement est effectué à la température ambiante et à la pression atmosphérique.

\section{CCM des tanins}

Pour l'identification des tanins, nous avons utilisé comme éluant un mélange d'Acétate d'Ethyle, de Méthanol et d'Eau dans les proportions $40 \mathrm{~mL}, 8 \mathrm{~mL}$ et $5 \mathrm{~mL}$. Pour cela, des plaques en verre recouvertes de gel de Silice sont utilisés comme phase stationnaire. La révélation est faite par une solution de chlorure ferrique après séchage. La coloration marron des spots est synonyme de la présence de tanins dans les extraits. $\mathrm{La}$ chromatographie est effectuée à une 
température d'environ $25{ }^{\circ} \mathrm{C}$ et à la pression atmosphérique.

\section{CCM des flavonoüdes}

Pour les flavonoïdes, l'éluant utilisé est un mélange d'Acétate d'Ethyle et d'Eau (15\%). La phase stationnaire a été des plaques en verre recouvertes de cellulose. La révélation est faite avec le Chlorure d'Aluminium et l'observation sous UV à 254 nm. La coloration jaune indique la présence de flavonoïdes. En marge de l'identification des flavonoïdes, pourrait se faire celle des polyphénols avec une révélation directe sous UV sans utilisation de réactifs. Ainsi, on pourrait observer plusieurs luminescences avec des colorations diverses à la température ambiante et à l'abri de la lumière.

\section{Identification des saponosides}

Pour rechercher les saponosides, $10 \mathrm{ml}$ de l'extrait total aqueux sont versés dans un tube à essais. Le tube est agité pendant $15 \mathrm{~s}$ puis laissé au repos durant $15 \mathrm{mn}$. Une hauteur de mousse persistante, supérieure à 1 $\mathrm{cm}$ indiquait la présence de saponosides.

\section{Test biologique}

Les tests concernent trois extraits (cyclohexanique, chloroformique et méthanolique) de Ficus thonningii et un insecte (Callosobrucus maculatus, ravageur du niébé) et une spéculation (niébé). Chaque extrait est testé sur l'insecte $C$. maculatus. A partir de chaque extrait sec, cinq solutions de dose différente $(1: 100 \mathrm{mg} / \mathrm{ml}, 2: 50 \mathrm{mg} / \mathrm{mL}$, 3: $25 \mathrm{mg} / \mathrm{mL}, 4: 12.5 \mathrm{mg} / \mathrm{mL}$ et $5: 6.25$ $\mathrm{mg} / \mathrm{mL}$ ) sont préparées pour voir l'effet de la dose sur la mortalité et l'émergence. La solution 5 est obtenue en prenant $1 \mathrm{~g}$ d'extrait sec que l'on dissout dans $10 \mathrm{ml}$ de solvant. La solution 4 est obtenue en pipetant $5 \mathrm{ml}$ de la solution 5 que l'on complète à $10 \mathrm{ml}$ avec du solvant. Avec le même procédé, nous avons obtenu la solution 3 à partir de la solution 4, 2 à partir du 3 et 1 à partir du 2 .

Les tests biologiques sont effectués dans des boîtes de pétri de diamètre $90 \mathrm{~mm}$. Dans chaque boîte, $20 \mathrm{~g}$ de niébé, $500 \mu \mathrm{l}$ de chaque solution et 25 insectes adultes de $C$. maculatus sont introduits. Le nombre de répétition pour chaque test est de quatre fois. Le tout est ensuite laissé à l'air libre pendant $20 \mathrm{mn}$ pour permettre l'évaporation du solvant. Au bout de 24 h, les tris ont débuté. Les nombres d'insectes morts, survivants et émergés sont ensuite dénombrés. La formule d'Abbott : Mc = (Mo-Mt) / (100-Mt)*100 ; (avec Mc : mortalité calculée, Mo: mortalité observée et Mt: mortalité dans les lots témoins) est utilisée pour corriger la mortalité observée.

\section{Analyse statistique}

Les variables mesurées sont les nombres d'insectes morts, survivants et émergés. Ces variables sont soumises à une analyse de variance, modèle fixe à trois facteurs (extraits, doses et temps). La variable taux de mortalité a subi une transformation $\arcsin (\mathrm{x}=$ taux de mortalité, $\mathrm{n}=$ taille de la population ; $n=1999$ ) afin de normaliser la population et de stabiliser la variance. La méthode General Linear Model dans Minitab 17 a été utilisée pour l'analyse statistique des données collectées. Les variables nombre d'insectes survivants et nombre d'insectes émergés quant à elles ont subi une transformation racine carré afin de normaliser la population et de stabiliser la variance. Les courbes et les tableaux sont utilisés pour présenter le résultat des analyses.

\section{RESULTATS}

\section{Etude phytochimique}

Les résultats des tests phytochimiques sont présentés dans le Tableau 1. Le tableau 1 des tests phytochimiques montre la présence d'alcaloïdes dans les extraits cyclohéxanique et chloroformique de la plante. Les flavonoïdes ne sont présents que dans l'extrait méthanolique. Les trois extraits cyclohéxanique, chloroformique et méthanolique contiennent tous des polyphénols. Des tanins sont identifiés au niveau des extraits chloroformique et méthanolique. La recherche de saponosides est positive que dans l'extrait aqueux de Ficus thonningii. Les photos de CCM des 
différentes familles illustrent bien les résultats des tests phytochimiques (Photo 1).

\section{Les tests biologiques}

L'analyse de la variance (Tableau 2) correspondant à l'effet insecticide montre que la mortalité a une variation hautement significative et significative respectivement suivant le temps et les extraits $(\mathrm{P}<0,001$; $\mathrm{P}<0,05)$. De même, les interactions tempsextraits sont significatives $(\mathrm{P}<0,05)$. Le facteur dose n'est pas significatif $(\mathrm{P}>0,05)$. Ce qui montre que l'effet insecticide dépend du temps et des extraits.

Les Figures 1 et 2 montrent l'évolution du traitement en fonction du temps, des doses et des extraits. Les résultats montrent aussi que l'extrait au méthanol (EM) est plus efficace sur Callosobruchus maculatus. Les extraits au Cyclohexane (EC) et au Chloroforme (EH) sont moyennement efficaces sur l'insecte. La courbe de mortalité en fonction du temps montre que le nombre d'insectes morts est plus élevé aux $3^{\text {ème }}$ jour, $5^{\mathrm{e}}$ jour, $6^{\mathrm{e}}$ jour, $7^{\mathrm{e}}$ jour et $8^{\text {ème }}$ jour.

Les Figures 3 et 4 donnent l'allure de la courbe des insectes émergés en fonction du temps, des doses appliquées et des extraits. Ces figures montrent que l'extrait méthanolique assure une meilleure protection du milieu vis-à-vis de Callosobruchus maculatus $\left(15^{\mathrm{e}}\right.$ jour, $16^{\mathrm{e}}$ jour, $20^{\mathrm{e}}$ jour et $22^{\mathrm{e}}$ jour).

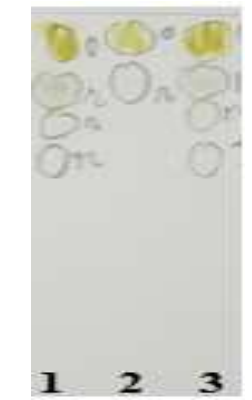

Photo CCM polyphénols

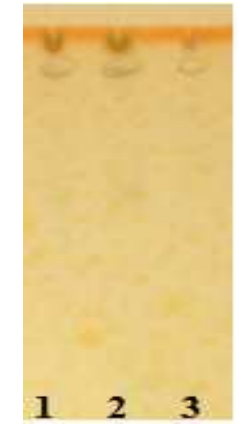

Photo CCM alcaloïdes

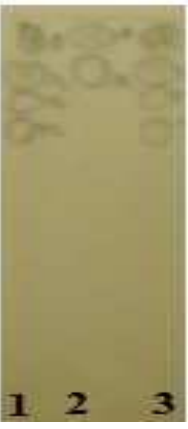

Photo CCM tanin

1 : extrait cyclohexanique, 2 : extrait chloroformique, 3 : extrait méthanoliqure.

Photo 1 : Les photos de CCM des différentes familles.

Tableau 1: Résultats des tests phytochimiques.

\begin{tabular}{lccccc}
\hline Extraits & Alcaloïdes & flavonoïdes & Polyphénols & Tanins & Saponosides \\
\hline Cyclohexanique & + & - & + & - & - \\
Chloroformique & + & + & + & + & - \\
Méthanolique & - & + & - & + & + \\
Aqueux & - & - & & & - \\
\hline
\end{tabular}


Tableau 2 : Résultats des traitements avec les extraits de Ficus thonningii sur Callosobruchus maculatus.

\begin{tabular}{lcccccc}
\hline \multirow{2}{*}{ Source de variation } & \multicolumn{3}{c}{ Mortalité } & \multicolumn{3}{c}{ Emergence } \\
\cline { 2 - 7 } & DL & F & P & DL & F & P \\
\hline Temps & 1 & 77,85 & 0,000 & 1 & 151,22 & 0,000 \\
Doses & 4 & 0,54 & 0,709 & 4 & 0,66 & 0,619 \\
Extraits & 2 & 4,27 & 0,014 & 2 & 27,78 & 0,000 \\
Temps doses & 4 & 0,30 & 0,880 & 4 & 0,71 & 0,588 \\
Temps extraits & 2 & 4,46 & 0,012 & 2 & 25,75 & 0,000 \\
Doses extraits & 8 & 1,20 & 0,298 & 8 & 1,75 & 0,092 \\
Temps doses extraits & 8 & 0,91 & 0,506 & 8 & 1,85 & 0,073 \\
\hline Erreur & 670 & & & 120 & & \\
Total & 699 & & & 159 & & \\
\hline
\end{tabular}

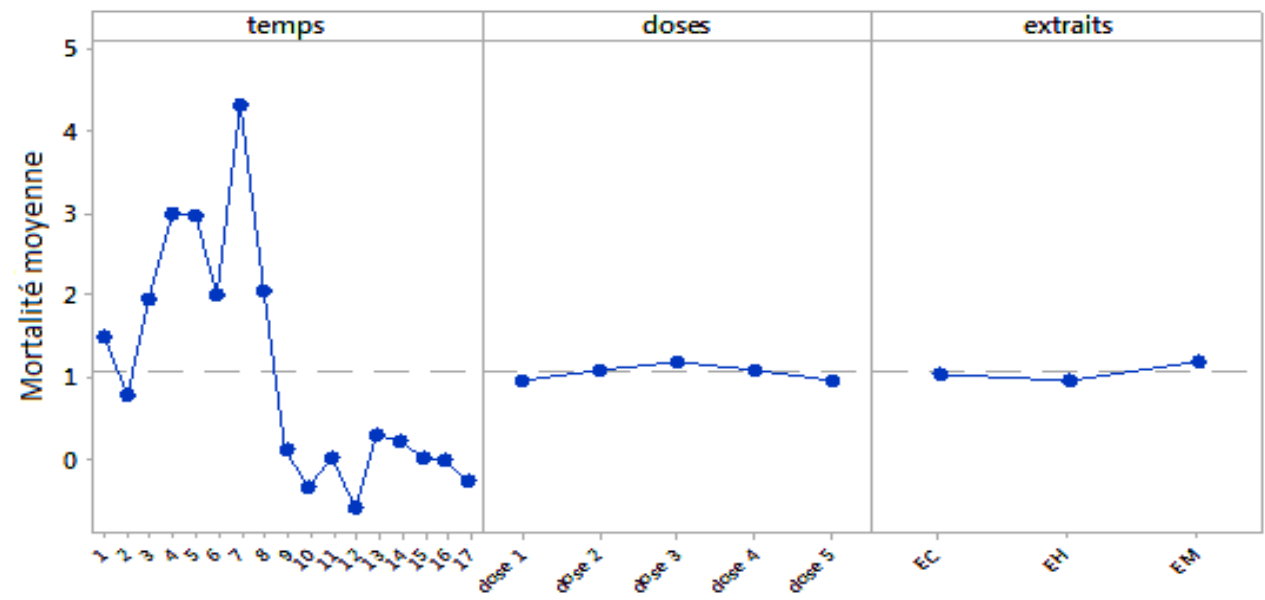

Figure 1 : Courbe de mortalité en fonction des extraits, doses, insectes et temps de Ficus thonningii. Dose $1=100 \mathrm{mg} / \mathrm{ml}$; dose $2=50 \mathrm{mg} / \mathrm{ml}$; dose $3=25 \mathrm{mg} / \mathrm{ml}$; dose $4=12.5 \mathrm{mg} / \mathrm{ml}$; dose $5=6.25 \mathrm{mg} / \mathrm{ml}$; EC: extrait cyclohexanique, EH: extrait chloroformique, EM: extrait méthanolique.

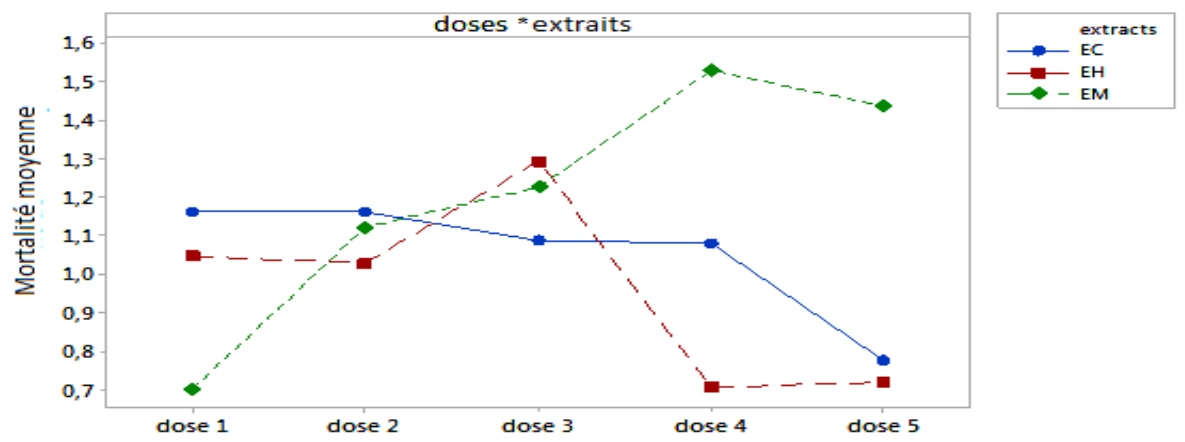

Figure 2 : Interaction entre extraits et doses de Ficus thonningii pour la mortalité. 


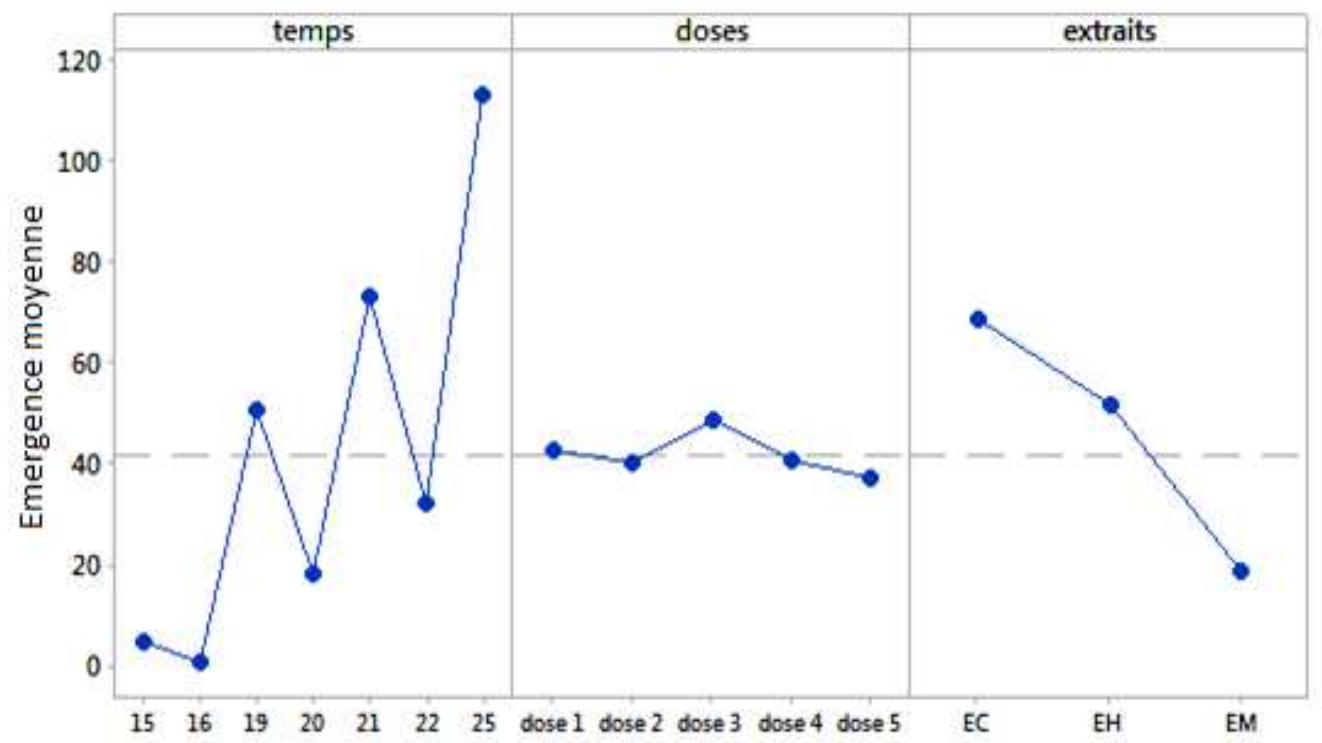

Figure 3 : Nombre d'insectes émergés en fonction des extraits, des doses et du temps.

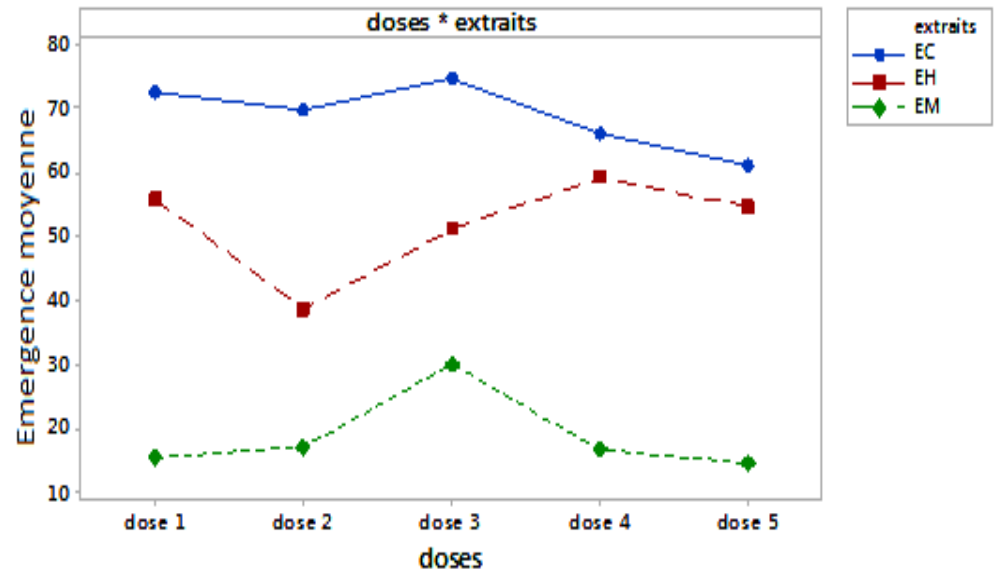

Figure 4 : Interaction entre extraits et doses de Ficus thonningii pour le nombre d'insectes émergés.

\section{DISCUSSION}

L'étude phytochimique montre que la plante contient des flavonoïdes, des tanins, des alcaloïdes et des saponosides. Plusieurs études antérieures sont confirmées par ces résultats. Parmi ces études, on peut citer celles de Ndukwe et al. (2007) qui ont montré la présence de carbohydrates, de glycosides, de saponines et d'alcaloïdes dans l'extrait méthanolique des feuilles, des tiges et des racines de Ficus thonningii. Les études de Onwkaeme et Udoh (2000) mettent en évidence la présence de tanins, de flavonoïdes, de saponines, d'anthraquinone et de glycosides dans les feuilles de Ficus thonningii. Ces études corroborent les résultats des tests phytochimiques effectués dans cette présente étude. La présence de ces métabolites secondaires pourrait expliquer ultérieurement les activités insecticides de la plante Ficus thonningii.

Par ailleurs, l'analyse statistique montre que l'extrait méthanolique donne un meilleur taux de mortalité sur Callosobruchus 
maculatus. Cela peut être expliqué par la présence dans cet extrait de certains métabolites secondaires tels que les polyphénols, les alcaloïdes et les tanins (Tableau 1) dont leurs toxicités vis-à-vis des insectes sont montrées dans plusieurs travaux. Parmi ces études, on peut citer :

Les travaux de Regnault-Roger et al., (2008) qui montrent que les polyphénols ubiquitaires dans la nature, provoquent une perturbation de la motricité naturelle de l'insecte. Celle-ci peut être rapide: dès le premier jour pour la quercétine, ou plus tardive, le quatrième jour, pour la narangine, le syringaldéhyde ou l'acide vanillique. Elle s'accompagne dans certain cas (acides caféique et férulique, vanilline, lutéoline 7 glucoside) d'un effet knock down. Au bout de huit jours, tous les insectes sont dans un état comateux ou morts. La toxicité des polyphénols est corrolée positivement au pouvoir attractif du composé.

Par ailleurs, les tanins présentent un effet direct toxique pour certaines espèces d'insectes (Raymond et al., 2011). Les tanins influencent sur la croissance, le développement et la fécondité de plusieurs insectes ravageurs (Vandenborre et al., 2011). La croissance réduite causée par les tanins a des inconvénients majeurs pour l'insecte, avec un plus faible nombre d'œufs et une plus petite taille d'œufs, ce qui affecterait la survie et la santé des individus de la génération subséquente (Meric, 2005). Ainsi, les plantes aromatiques et leurs molécules allélochimiques exercent une double activité : - sur les adultes : par une action toxique rapide de type inhalatoire (monoterpènes) d'une part, et par action, qui concourt à l'activité insecticide de la plante aromatique, d'une intensité moindre mais qui s'exerce dans la durée (polyphénols) ;

- sur les différentes phases du cycle reproductif : inhibition de la fécondité, activité ovocide et larvicide aux stades néonatal et ultérieurs.

Ficus thonningii fait parti des Angiospermes. Ce qui pourrait expliquer ses effets insecticides car les Angiospermes contiennent des alcaloïdes qui sont des métabolites secondaires constitués des atomes d'azote secondaire, tertiaire ou quaternaire dans leurs structures (Bruneton, 2014). Ils sont métaboliquement actifs et jouent un rôle important dans la physiologie des plantes ou des organismes. Les alcaloïdes possèdent des propriétés répulsives ou anti-appétantes à l'égard des insectes ravageurs (Pelletier, 2001).

Beaucoup de travaux ont montré aussi que les extraits organiques de plante donnent des effets insecticides sur les insectes ravageurs de denrées stockées. La toxicité des extraits aux solvants organiques de Afrostyrax lepidophilus, de Trichilia gilgiana, de Drypetes gossweileri et de Zanha golungensis à l'égard de Sitophilus zeamaïs, de Tribolium castaneum et de Rhyzopertha dominica est montrée par les travaux de Toumnou (2013).

\section{Conclusion}

Les tests insecticides des trois extraits (cyclohexanique, chloroformique et méthanolique) de Ficus thonningii montrent que l'extrait méthanolique donne un meilleur taux de mortalité sur Callosobruchus maculatus. Ce résultat est confirmé par l'étude phytochimique. Cet extrait sera soumis à un fractionnement et la purification afin d'isoler le ou les principe (s) actif (s).

\section{REFERENCES}

Toumnou A. 2013. Integrated management of the principal devastating insects of cereals by the use of the secondary metabolites of the indigenous plants of Senegal and Central Africa. Doctorate, SEV, UCAD.

Bottenberg H. 1995. Farmers perception of crop pest control practices I rainfed cowpea cropping systems in Kano, Nigeria. Inter. J. Pest Management, 41(4): 195-200.

Bruneton J. 2014. PharmacognosiePhytochimie, Plantes Médicinales $\left(6^{\mathrm{e}}\right.$ édn, revue et augmentée). Tec \& Doc: Paris; 1288.

CGIAR. 2001. Cowpea (Vigna unguiculata). CGIAR on line/CGIAR Research: Areas of Research, <http:/www.cgiar.org/ research/res_cowpea.html>, Washington, D.C. 
Cissé N, Hall AE. 2003. Traditional Cowpea in Senegal, a case study. 27p. www.fao.org/ag/AGP/AGPC/doc/publica t/cowpea_Cisse/cowpea_cisse_e.htm.

Onwkaeme DN, Udoh F. 2000. Pharmacognostic and anti-diarrhoeal studies of leaves of Ficus thonningii. Nig. J. Nat Prod. And Med., 4: 27-29.

DSDIA/DAPS/MAE. 2003. Résultats définitifs des récoltes des campagnes agricoles 1998/1999 à 2002/2003. Récapitulatif des cultures industrielles et autres cultures. Sénégal, $3^{\text {ème }}$ version du 24/03/2003.

Bassène E. 2014. Extractions et Lipides. UCAD, p8.

F.A.O. 1998. La situation mondiale de l'alimentation et de l'agriculture, F.A.O. $371 \mathrm{p}$.

FAOSTAT. 2004. Agricultural Production, Crop Primary Database. Food and Agricultural Organisation of the United Nations: Rome.

IITA. 2009. Production du niébé en Afrique de l'Ouest: Guide du paysan. www.iita.org, 26.

Djossou J. 2006. Etude des possibilités d'utilisations des formulations à base de fruits secs de Xylopia aethiopica Dunal (Annonaceae) pour la protection des stocks de niébé contre Callosobruchus maculatus Fabricius (Coleoptera : Bruchidae). Faculté des Sciences Agronomiques de Gembloux Belgique.

Ngamo LST, Hance Th. 2007. Diversité des ravageurs des denrées et méthodes alternatives de lutte en milieu tropical. Tropicultura, 4: 215-220.

Meric K. 2005. Studies on the compounds polyphenolic in relation to the food of the tortrix of the buds of the virginal (Choristoneura fumiferana (Clem.). Doctorate, Sciences Forest, Univ. Laval.

Bouzouita N, Kachouri F, Ben Halima M, Chaabouni MM. 2008. Composition chimique et activités antioxydante, antimicrobienne et insecticide de l'huile essentielle de Juniperus phoenicea. Journal de la Société Chimique de Tunisie, 10: 119-125.

Ndukwe IG, Bello AI, Habila JD, John C. 2007. Phytochemical and antimicrobial screening of the crude petroleum spirit and methanol extracts of the stem bark, leaves and roots of Ficus thoningii (blume). African Journal of Biotechnology, 6(23): 2645-2649.

Nkouannessi M. 2005. The genetic, morphological and physiological evaluation of African cowpea genotypes. Thesis, University of the Free State Bloemfontein, South Africa, 131p.

Diouf GEl-H, Samb A, Seck D, Diop M. 2014. Phytochimical and insecticidal study of three organic extracts of Crataeva religiosa Forst on Sitophilus zeamais and Callosobruchus maculatus. Int. Res J Pharm. App Sci., 4(4):13-18.

Singh S, Van Emden HF. 1979. Insect pests of grain legumes. Ann. Rev. Entomol., 24: 255-278.

Vandenborre G, Smagghe G, Van Dammea JM. 2011. Plant lectins as defense proteins against phytophagous insects. Phytochemistry, 72: 1538-1550. 